\title{
Infecciones del sitio operatorio posterior a mastectomía radical modificada, análisis epidemiológico en una clínica oncológica
}

\author{
Surgical site infections after modified radical mastectomy, epidemiological \\ analysis at an oncology center
}

\author{
Mara García $^{1} \mathbb{D}$, Estephannia Mora² ${ }^{\mathbb{D}}$, Alicia Maza ${ }^{3}$, Jorge Hernán Hoyos ${ }^{3}$
}

1 Médica infectóloga, Servicio de Infectología y Epidemiología Hospitalaria, Instituto Médico de Alta Tecnología, IMAT Oncomédica, Montería, Colombia

2 Odontóloga, epidemióloga, Servicio de Infectología y Epidemiología Hospitalaria, Instituto Médico de Alta Tecnología, IMAT Oncomédica, Montería, Colombia

3 Médico, Servicio de Infectología y Epidemiología Hospitalaria, Instituto Médico de Alta Tecnología, IMAT Oncomédica, Montería, Colombia

\section{Resumen}

Introducción. La tasa de incidencia de infecciones del sitio operatorio asociadas a cirugías mamarias varía desde el 6 hasta el 38 \%. Se presentan la incidencia local y el perfil microbiológico de las infecciones en una clínica oncológica.

Métodos. Se trata de un estudio de cohorte, prospectivo, descriptivo, durante un periodo de un año en el Instituto Médico de Alta Tecnología, IMAT Oncomédica, de mujeres con diagnóstico de cáncer de mama, que presentaron infecciones del sitio operatorio después de la mastectomía.

Resultados. Se encontraron 335 cirugías registradas y la incidencia de infecciones del sitio operatorio fue del $3,38 \%$, todas en mastectomías radicales con reconstrucción. Se obtuvo crecimiento bacteriano en el $77 \%$ de los cultivos, principalmente de cocos Gram positivos, con predominio de Staphylococcus aureus sensible a la meticilina (SAMS). Los bacilos Gram negativos representaron el 40 \%. Se administraron cefalosporinas de primera generación como profilaxis antibiótica prequirúrgica, la cual fue correcta en el 3I \% de los casos. En el 50 \% de las pacientes infectadas se practicó el baño prequirúrgico y se cumplió el protocolo institucional.

Conclusiones. La incidencia encontrada de infecciones del sitio operatorio es menor que la reportada en otras series. Staphylococcus aureus sensible a la meticilina fue el microorganismo responsable más frecuente. El cumplimiento de la profilaxis con cefalosporina y baño prequirúrgico es fundamental para disminuir la incidencia de infecciones del sitio operatorio.

Palabras clave: neoplasias de la mama; microbiología; mastectomía radical modificada; infección de la herida quirúrgica.

Fecha de recibido: 30/09/2019 - Fecha de aceptación: 24/10/2019

Correspondencia: Estephannia Mora, Carrera 6 N 72-34, Montería, Colombia. Teléfono: 785-4344

Correo electrónico: mora.investigación@gmail.com

Citar como: García M, Mora E, Maza A, Hoyos JH. Infecciones del sitio operatorio posterior a mastectomía radical modificada, análisis epidemiológico en una clínica oncológica. Rev Colomb Cir. 2020;35:398-403. https://doi.org/10.30944/20117582.466

Este es un artículo de acceso abierto bajo una Licencia Creative Commons - BY-NC-ND https://creativecommons.org/licenses/by-ncnd/4.0/deed.es 


\begin{abstract}
Introduction. The incidence rate of surgical site infection associated with breast surgeries ranges from $6 \%$ to $38 \%$. We present the local incidence and microbiological profile of infections in an oncology clinic.

Methods. Prospective, descriptive cohort study over a one-year period of women diagnosed with breast cancer at Instituto Médico de Alta Tecnología, IMAT Oncomédica, and have developed surgical site infection after mastectomy.

Results. 335 registered surgeries were found. The incidence of surgical site infection was $3.38 \%$, all of them in radical mastectomies with reconstruction. Bacterial growth was obtained in $77 \%$ of crops. Gram-positive were the main microorganisms responsible, predominantly methicillin-sensitive Staphylococcus aureus (SAMS). Gram-Negative bacilli accounted for $40 \%$. First generation cephalosporins were administered as presurgical antibiotic prophylaxis, which was correct in $31 \%$ of cases. In $50 \%$ of infected patients, the pre-surgical bath was performed and the institutional protocol was complied with.
\end{abstract}

Conclusion. Our incidence of surgical site infection was lower than reported in other series. SAMS was the most common responsible microorganism. Compliance with cephalosporin and pre-surgical bath prophylaxis is critical to reducing the incidence of operative site infections.

Keywords: breast neoplasms; microbiology; mastectomy, modified radical; surgical wound infection.

\section{Introducción}

Aproximadamente, de 187 a 28I millones de procedimientos quirúrgicos se realizan anualmente en todo el mundo. Las infecciones del sitio operatorio son las infecciones hospitalarias más comunes y corresponden al $38 \%$ del total; de estas infecciones, dos tercios están confinados a la incisión y un tercio corresponde a los órganos y espacios involucrados durante la cirugía ${ }^{\text {. }}$. En múltiples investigaciones se ha demostrado que las infecciones del sitio operatorio generan un aumento de los días de estancia hospitalaria y, por ende, de los costos hospitalarios ${ }^{2-4}$. Se estima que más de la mitad son prevenibles con la aplicación de medidas basadas en la evidencia científica. La mayoría de las infecciones del sitio operatorio se originan durante el procedimiento mismo. Después de la cirugía, se producen pocas infecciones si se ha hecho el cierre primario de la herida 5 .

El primer reservorio de microorganismos que causan infecciones del sitio operatorio es la flora endógena del paciente, que contamina la herida por contacto directo. Por esta razón, la preparación del paciente debe ser meticulosa, con el objeto de disminuir su carga microbiana en el intestino, la piel o el sistema genital, según el procedimiento al que sea sometido. En lo que se refiere a procedimientos quirúrgicos limpios, la contaminación exógena de las heridas es determinante. El personal de cirugía constituye la fuente primaria de agentes patógenos aéreos en el quirófano y esto se debe a la difusión de una gran cantidad de microorganismos de la piel.

La incidencia de infecciones asociadas a cirugías mamarias varía ampliamente en la literatura científica y, según el tipo de cirugía y de paciente, se reportan tasas que van desde el 6 hasta el $38 \%{ }^{6,7}$. La cirugía mamaria se considera un procedimiento limpio, por lo tanto, el uso de la profilaxis antibiótica en ausencia de factores de riesgo de infección es discutido. Los organismos más comunes son Staphylococcus aureus, otros estafilococos y estreptococos. En situaciones que involucran tejidos macerados y húmedos (región axilar) y en pacientes con diabetes y obesidad, se ha reportado una mayor tasa de infecciones del sitio operatorio por gérmenes Gram negativos ${ }^{8}$.

Este estudio tuvo como objetivo conocer la incidencia local y el perfil microbiológico de las infecciones después de la mastectomía radical modificada en una clínica oncológica. 


\section{Métodos}

Se llevó a cabo un estudio de cohorte, prospectivo y descriptivo, de mujeres con cáncer de mama que fueron sometidas a mastectomía radical modificada, con reconstrucción o sin ella, durante el periodo comprendido entre enero de $2018 \mathrm{y}$ enero de 2019 en el Instituto Médico de Alta Tecnología (IMAT Oncomédica) de Montería, Córdoba.

En dicho Instituto, se elaboró una guía con recomendaciones basadas en la prevención de la infección del sitio operatorio, las cuales se encuentran divididas en preoperatorias, intraoperatorias y posoperatorias. Para el periodo preoperatorio, se diseñó un folleto ilustrativo y educativo, que se le entrega al paciente durante la programación de la cirugía, en el que se menciona la importancia del baño prequirúrgico, el cual está indicado en todos los procedimientos. Se recomienda un jabón neutro para las cirugías de menor riesgo y jabón con gluconato de clorhexidina para las cirugías de mayor riesgo (cirugías cardiacas, prótesis articulares y neurocirugías). El baño debe iniciarse en las partes menos contaminadas y continuar hacia las más contaminadas, como cabello, brazos, torso, espalda, piernas y zona perineal. Se recomienda un baño en la noche de la víspera de la cirugía y, otro, el día del procedimiento. En cirugías de alto riesgo, se inicia desde 72 horas antes del procedimiento. Se aclara la importancia de retirar todos los accesorios (anillos, reloj, pulseras, aretes), cortarse las uñas, no afeitarse en el domicilio y retirar el esmalte de las uñas.

La profilaxis antibiótica se hizo con cefalosporinas de primera generación (cefazolina o cefalotina) según la indicación de la guía institucional. Se consideró correcta la profilaxis antibiótica cuando se administró 30 a 60 minutos antes de la incisión quirúrgica, a dosis de $2 \mathrm{~g}$ por vía intravenosa.

El seguimiento posoperatorio estuvo a cargo del equipo de cirugía mamaria y del Servicio de Infectología. Las citas médicas de control fueron a los 7, I4 y 30 días después de la cirugía. En el seguimiento se incluyeron los siguientes aspectos: evaluación médica, cicatrización de heridas, uso de antibióticos y dispositivo de drenaje utilizado (en días).

Para el diagnóstico de infección del sitio operatorio, se tuvieron en cuenta los siguientes criterios: drenaje de material purulento por la incisión superficial; dolor, hipersensibilidad, edema y enrojecimiento o calor local asociado o no con dehiscencia de suturas, y, aislamiento de microorganismos en el cultivo del líquido o tejido obtenido asépticamente. La infección en los primeros 30 días del posoperatorio se consideró temprana y, después de los 30 días, tardía.

Los datos recolectados se obtuvieron de las historias clínicas de los pacientes y, posteriormente, la información se registró en una base de datos de Microsoft Excel y se analizó en el software R. Los resultados se presentan en frecuencias absolutas y porcentajes. La asociación entre las variables y el tipo de cirugía se evaluó mediante el test exacto de Fisher.

\section{Resultados}

Durante el periodo comprendido entre enero de 2018 y enero de 2019 en el Instituto Médico de Alta Tecnología (IMAT Oncomédica) de Montería, se practicaron 335 procedimientos de mastectomía radical modificada. La incidencia de infecciones del sitio operatorio fue de 13 casos, es decir, $3,38 \%$. Se obtuvo crecimiento bacteriano en el $77 \%$ de las muestras enviadas para cultivo. Los cocos Gram positivos fueron los principales microorganismos responsables y, entre ellos, Staphylococcus aureus sensible a la oxacilina fue el más frecuente (46\%). En $40 \%$ de los casos se aislaron bacilos Gram negativos y, entre estos, Klebsiella pneumoniae multisensible fue el principal. Hubo una mayor incidencia de infección por $S$. aureus en las pacientes sometidas a mastectomía radical modificada con vaciamiento axilar, que en aquellas sometidas a otros procedimientos (tabla I).

$\mathrm{El} 50 \%$ de las pacientes infectadas afirmaron haber cumplido con el baño prequirúrgico y con las recomendaciones del protocolo institucional. Para la profilaxis antibiótica prequirúrgica 
Tabla 1. Pacientes con infección del sitio operatorio después de mastectomía radical modificada

\begin{tabular}{|c|c|c|c|c|}
\hline \multicolumn{2}{|c|}{ Variables } & \multirow{2}{*}{$\begin{array}{c}\text { MRM + colgajo } \\
\mathbf{n}(\%)\end{array}$} & \multirow{2}{*}{$\begin{array}{c}\text { MRM + colgajo + VAC } \\
\mathbf{n}(\%)\end{array}$} & \multirow{2}{*}{$\begin{array}{c}\text { MRM + VAC + BC } \\
\mathbf{n}(\%) \\
1(7,7) \\
\end{array}$} \\
\hline & Pacientes & & & \\
\hline \multirow{2}{*}{ Profilaxis antibiótica correcta } & No & $5(38,5)$ & $4(53,9)$ & $1(7,7)$ \\
\hline & Sí & $2(15,4)$ & $1(15,4)$ & $0(0,0)$ \\
\hline \multirow{2}{*}{ Medicamento de profilaxis } & Cefalotina & $3(23,1)$ & $4(30,8)$ & $1(7,7)$ \\
\hline & Cefazolina & $0(0,0)$ & $5(38,5)$ & $0(0,0)$ \\
\hline \multirow{2}{*}{ Drenaje Hemovac } & $>7$ días & $3(23,1)$ & $9(69,2)$ & $0(0,0)$ \\
\hline & Sí & $0(0,0)$ & $0(0,0)$ & $1(7,7)$ \\
\hline \multirow{3}{*}{ Baño prequirúrgico } & Desconocido & $0(0,0)$ & $2(15,4)$ & $0(0,0)$ \\
\hline & No & $2(15,4)$ & $3(23,1)$ & $0(0,0)$ \\
\hline & Sí & $1(7,7)$ & $4(53,9)$ & $1(7,7)$ \\
\hline \multirow{6}{*}{ Germen aislado } & $\begin{array}{l}\text { Klebsiella } \\
\text { pneumoniae }\end{array}$ & $2(15,4)$ & $0(0,0)$ & $0(0,0)$ \\
\hline & Negativo & $1(7,7)$ & $2(15,4)$ & $0(0,0)$ \\
\hline & Proteus mirabilis & $0(0,0)$ & $1(7,7)$ & $0(0,0)$ \\
\hline & $\begin{array}{l}\text { Staphylococcus } \\
\text { aureus }\end{array}$ & $0(0,0)$ & $5(38,5)$ & $0(0,0)$ \\
\hline & $\begin{array}{l}\text { Streptococus } \\
\text { pyogenes }\end{array}$ & $0(0,0)$ & $0(0,0)$ & $1(7,7)$ \\
\hline & Serratia marscecens & $0(0,0)$ & $1(7,7)$ & $0(0,0)$ \\
\hline \multirow{3}{*}{$\begin{array}{l}\text { Sensibilidad a la } \\
\text { cefalosporina de primera } \\
\text { generación }\end{array}$} & No aplica & $1(7,7)$ & $2(15,4)$ & $0(0,0)$ \\
\hline & No & $0(0,0)$ & $2(15,4)$ & $0(0,0)$ \\
\hline & Sí & $2(15,4)$ & $5(38,5)$ & $1(7,7)$ \\
\hline \multirow{2}{*}{ Quimioterapia previa } & No & $2(15,4)$ & $3(23,1)$ & $0(0,0)$ \\
\hline & Sí & $1(7,7)$ & $6(46,2)$ & $1(7,7)$ \\
\hline \multirow{2}{*}{ Radioterapia previa } & No & $2(15,4)$ & $9(69,2)$ & $1(7,7)$ \\
\hline & Sí & $1(7,7)$ & $0(0,0)$ & $0(0,0)$ \\
\hline \multirow{2}{*}{$\begin{array}{l}\text { Clasificación de la ISO según } \\
\text { el tiempo de evolución }\end{array}$} & Tardía & $1(7,7)$ & $1(7,7)$ & $1(7,7)$ \\
\hline & Temprana & $2(15,4)$ & $8(61,5)$ & $0(0,0)$ \\
\hline \multirow{2}{*}{$\begin{array}{l}\text { Días para el diagnóstico } \\
\text { de infección }\end{array}$} & Promedio de días & 20,7 & 19,9 & 32,0 \\
\hline & Desviación en días & 10,8 & 8,7 & NA \\
\hline
\end{tabular}

ISO: infección del sitio operatorio; MRM: mastectomía radical modificada; VAC: vaciamiento ganglionar axilar; BC: biopsia de ganglio centinela

se usaron cefalosporinas de primera generación (cefazolina) y su administración, en cuanto a dosis y tiempo, se consideró correcta en el 3I \% de los casos. Todas las pacientes infectadas permanecieron con un drenaje Hemovac durante más de siete días. El antimicrobiano más utilizado para tratar la infección fue trimetoprim-sulfametoxazol.

\section{Discusión}

Existen pocos estudios nacionales sobre la incidencia de las infecciones del sitio operatorio después de cirugías oncológicas mamarias; uno de ellos es el de Gil-Londoño, et al., en Medellín, Antioquia, en el cual se reportó una incidencia del $16 \%$. Por el contrario, en estudios internacionales se informan tasas que oscilan entre el 2,5y el $8 \%{ }^{\mathrm{ro}}$. La incidencia encontrada en el presente estudio es inferior a la de otras series.

Entre los factores asociados con las infecciones del sitio operatorio en cirugías mamarias oncológicas, se destacan la obesidad, la radioterapia, la colonización previa por $S$. aureus resistente a la oxacilina, el implante de prótesis y 
la duración prolongada del drenaje Hemovac. Algunos autores recomiendan el retiro del drenaje con un débito menor de $30 \mathrm{ml} /$ día, y otros sugieren su retiro con 30 a $50 \mathrm{ml}$ cada 48 horas o a los cinco a siete días ${ }^{\mathrm{II}, 12}$.

Los microorganismos más comunes en procedimientos considerados limpios son aquellos incluidos en la flora habitual de la piel de los pacientes: especies de estreptococos y estafilococos positivos o negativos para coagulasa ${ }^{\mathrm{I} 3}$. Según la literatura científica, $S$. aureus resistente a la oxacilina es el microorganismo más frecuente, por lo que se debe tener en cuenta en algunas ocasiones el eventual reemplazo de la profilaxis antibiótica con cefalosporinas de primera generación por antibióticos con espectro para S. aureus resistente a la oxacilina y Staphylococcus spp. negativo para la coagulasa ${ }^{8}$. Este estudio mostró mayor incidencia de $S$. aureus sensible a la oxacilina, lo cual sugiere continuar la profilaxis antibiótica con cefalosporinas de primera generación.

Los autores recomiendan el uso de la profilaxis antibiótica en todas las cirugías oncológicas mamarias, dejando a consideración de cada institución el esquema antibiótico según la epidemiología local. La profilaxis antimicrobiana previene las infecciones del sitio operatorio al reducir la carga de microorganismos en el sitio quirúrgico durante el procedimiento, y disminuye la duración y el costo de la atención médica ${ }^{14}$.

Para lograr estos objetivos, el agente antimicrobiano debe ser activo contra los agentes patógenos con mayores probabilidades de contaminar el sitio quirúrgico, debe administrarse en dosis única y en el momento apropiado para garantizar concentraciones adecuadas en el suero y los tejidos al momento de la incisión quirúrgica (periodo de posible contaminación). Avellaneda-Oviedo, et al., reportaron una tasa de cumplimiento del $60 \%{ }^{\text {II }}$. En la presente serie, la administración de la profilaxis fue correcta en el 3I \% de los casos, porcentaje que consideramos inferior al esperado, y que incentiva a evaluar y fortalecer el cumplimiento del protocolo de profilaxis antibiótica prequirúrgica con el Servicio de Cirugía de la Institución.

\section{Conclusión}

Las infecciones del sitio operatorio posteriores a las cirugías oncológicas mamarias en nuestra Institución, muestran tasas inferiores a las reportadas en otras instituciones del país. Staphylococcus aureus sensible a la oxacilina es el principal microorganismo responsable, lo cual incentiva a reforzar el cumplimiento de la profilaxis antibiótica con cefalosporinas de primera generación y el baño prequirúrgico en las pacientes.

\section{Cumplimiento de normas éticas}

Consentimiento informado. El Comité de Ética Institucional aprobó el diseño y la metodología del estudio. Se obtuvieron los consentimientos informados por parte de las pacientes.

Declaración de conflicto de intereses. Los autores declaran que no tienen conflicto de intereses.

Fuentes de financiación. Recursos propios de los autores.

\section{Referencias}

I. Garner J. The CDC Hospital Infection control practices advisory committee. Guideline for prevention of surgical site infection. Am J Infect control. 1993;27:97-I32.

2. Cruse P. Wound infection surveillance. Rev Infect Dis. I98I;4:734-7. https://doi.org/Io.I093/clinids/3.4.734

3. Cruse P, Ford R. The epidemiology of wound infection surveillance: A Io-year prospective study of 62,939 wounds. Surg Clin North Am. 1980;60:27-40. https://doi.org/Io.IoI6/So039-6IO9(I6)4203I-I

4. Martone W, Jarvis W, Culver D, Haley R. Incidence and nature of endemic nosocomial infections. En: Bennett J, Brachman P, editors. Hospital infections. Third edition. Boston: Little, Brown and Co.; 1992. p. 577-96.

5. Leaper D, Edmiston C. World Health Organization: global guidelines for the prevention of surgical site infection. J Hosp Infect. 20I7;95:135-6.

https://doi.org/IO.IoI6/j.jhin.20I6.I2.0I6

6. Chattha A, Cohen J, Bucknor A, Chen A, Tobias A, Lee $B$, Lin S. Surgical site infection in immediate breast reconstruction: Does chemotherapy timing make a difference? J Surg Oncol. 2018;II7:I440-6.

https://doi.org/IO.IOO2/jso.25053

7. Vilar-Compte D, Jacquemin B, Robles C, Volkow P. Surgical site infections in breast surgery: Case-control study. World J Surg. 2004;28:242-6.

https://doi.org/IO.IOO7/s00268-003-7193-3 
8. Hernaiz-Leonardo J, Golzarri M, Cornejo-Juárez P, Volkow P, Velázquez C, Ostrosky-Frid M, Vilar-Compte D. Microbiology of surgical site infections in patients with cancer: A 7-year review. Am J Infect Control. 20I7;45:76I-6. https://doi.org/Io.IoI6/j.ajic.2017.02.023

9. Gil-Londoño J, Nagles-Peláez J, Maya-Salazar W, Madrid J, Maya-Restrepo M, Agudelo-Pérez R, et al. Surgical site infection after breast cancer surgery at 30 days and associated factors. Infectio. 20I7;2I:96-IOI.

https://doi.org/IO.22354/in.v2ri2.653

Io. Caisley L, Clark K. Surgical site infection in breast cancer surgery: A single centre study. Eur J Surg Oncol. 2017;43:S23. https://doi.org/Io.IOI6/j.ejso.2017.0I.095

II. Avellaneda-Oviedo M, Fernández-Gómez F, Pacheco-Compaña J, Comellas-Melero N, Gutiérrez J, Mariñas J. Análisis de la utilización de un protocolo de profilaxis antibiótica en cirugía mamaria. Cir Plást
Iberolatinoam. 2016;42:255-64.

https://doi.org/I0.432I/So376-78922016000300007

I2. Gupta R, Pate K, Varshney S, Goddard J, Royle G. A comparison of 5-day and 8-day drainage following mastectomy and axillary clearance. Eur J Surg Oncol. 200I;27:26-30. https://doi.org/IO.IO53/ejso.2000.I054

I3. Hidron A, Edwards J, Patel J, Horan T, Sievert D, Pollock D, et al. Antimicrobial-resistant pathogens associated with healthcare-associated infections: Annual summary of data reported to the National Healthcare Safety Network at the Centers for Disease Control and Prevention, 2006-2007. Infect Control Hosp Epidemiol. 2008;29:996-IOII. https://doi.org/IO.IO86/59I86I

I4. Voit S, Todd J, Nelson B, Nyquist A. Electronic surveillance system for monitoring surgical antimicrobial prophylaxis. Pediatrics. 2005;II6:1317-22. https://doi.org/IO.I542/peds.2004-I969 